\title{
Reply to the letter by L. Czakó et al. regarding "Endoscopic submucosal dissection as a treatment for gastric noninvasive neoplasia: a multicenter study by Osaka University ESD Study Group”
}

\author{
Motohiko Kato $\cdot$ Tsutomu Nishida $\cdot$ \\ Tetsuo Takehara
}

Received: 11 November 2011 / Accepted: 12 December 2011/Published online: 11 January 2012

(C) Springer 2012

We thank Dr. László Czakó et al. for their letter and interest regarding our article [1]. Dr. Czakó et al. made the following points concerning the strategy for treating gastric non-invasive neoplasia (NIN): (1) gastric NIN should be endoscopically resected; (2) concerning the modality of the treatment, endoscopic mucosal resection (EMR) may be recommended for the removal of small $(<5 \mathrm{~mm})$, elevated lesions, and endoscopic submucosal dissection (ESD) would be preferred for larger or depressed lesions.

The concept of adenoma-carcinoma sequence has not been fully applied in the carcinogenesis of gastric cancer compared with colorectal cancer. Therefore, there are some controversies whether endoscopic resection is needed for low-grade gastric NIN, but endoscopic resection is acceptable for high-grade gastric NIN as well as early gastric cancer. Our study, which included patients with both low- and high-grade NIN, showed that $44 \%$ of the patients were diagnosed as having cancer after ESD. Moreover, another group reported that about $34 \%$ of gastric low-grade NINs (Vienna classification category 3) were diagnosed as high-grade NIN (category 4) or invasive cancer (category 5) [2]. These results are based on retrospective study and focused on the patients who underwent endoscopic resection; therefore, the incidence rate of gastric cancer might be overestimated. However, it would have been preferable if both high- and low-grade gastric NIN had been the therapeutic target for endoscopic treatment. We cannot make a conclusion from this study whether all NINs need to be resected and which modality (conventional EMR or ESD) is optimal due to the limitations of our study.

In order to validate endoscopic resection for low-grade gastric NIN, we are planning a multicenter prospective study to clarify the incidence rate of gastric cancer after endoscopic resection (both conventional EMR and ESD). We will then evaluate the efficacy of each endoscopic modality from the viewpoints of curability, safety, and economic aspects in the future.

\section{References}

1. Kato M, Nishida T, Tsutsui S, Komori M, Michida T, Yamamoto $\mathrm{K}$, et al. Endoscopic submucosal dissection as a treatment for gastric noninvasive neoplasia: a multicenter study by Osaka University ESD Study Group. J Gastroenterol. 2011;46:325-31.

2. Cho SJ, Choi IJ, Kim CG, Lee JY, Kook MC, Park S, et al. Risk of high-grade dysplasia or carcinoma in gastric biopsy-proven lowgrade dysplasia: an analysis using the Vienna classification. Endoscopy. 2011;43:465-71.

This author's reply refers to the letter to the editor at doi:10.1007/s00535-011-0525-4.

M. Kato $(\square) \cdot$ T. Nishida $\cdot$ T. Takehara

Department of Gastroenterology and Hepatology, Osaka

University Graduate School of Medicine, 2-2 Yamadaoka, Suita,

Osaka 565-0871, Japan

e-mail: motohiko@gh.med.osaka-u.ac.jp 